\title{
Sensitivity of $D$ meson azimuthal anisotropies to system size and nuclear structure
}

\section{Roland Katz}

SUBATECH, Université de Nantes, EMN, IN2P3/CNRS, 44307 Nantes, France

\section{Caio A. G. Prado}

Institute of Particle Physics, Central China Normal University (CCNU), Wuhan, Hubei 430079, China

\section{Jacquelyn Noronha-Hostler*}

University of Illinois at Urbana-Champaign, Urbana, IL 61801, United States

Department of Physics and Astronomy, Rutgers University, Piscataway, NJ 08854, USA

jnorhoseillinois.edu

\section{Jorge Noronha}

Instituto de Física, Universidade de São Paulo, C.P. 66318, 05315-970 São Paulo, SP, Brazil

\author{
Alexandre A. P. Suaide \\ Instituto de Física, Universidade de São Paulo, C.P. 66318, 05315-970 São Paulo, SP, Brazil
}

\section{Marcelo G. Munhoz}

Instituto de Física, Universidade de São Paulo, C.P. 66318, 05315-970 São Paulo, SP, Brazil

\begin{abstract}
Recent experimental data at the Large Hadron Collider (LHC) confirmed that the soft sector azimuthal anisotropies in central XeXe collisions are sensitive to deformations in the wave function of the Xenon nucleus. Additionally, the CMS experiment found that D meson flow is slightly suppressed compared to other particle species when considering quark number scaling in small systems of $\mathrm{pPb}$ compared to $\mathrm{PbPb}$. In this talk we used the $\mathrm{D}$ and $\mathrm{B}$ Mesons Modular (DABMOD) code coupled to Trento+v-USPhydro to calculate the $R_{A A}$ and $v_{n}$ 's of D mesons using different energy loss models and Langevin techniques. Comparing D mesons $R_{A A}$ and $v_{n}$ in $\mathrm{PbPb}$ to XeXe collisions it is found that in central collisions D meson azimuthal anisotropies are sensitive to details in the nuclear structure wave function of Xenon. Additionally, we find that in mid-central collisions the smaller system size of XeXe suppresses D meson flow.
\end{abstract}

13th International Workshop in High pT Physics in the RHIC and LHC Era (High-pT2019)

19-22 March 2019

Knoxville, Tennessee, USA

${ }^{*}$ Speaker. 


\section{Introduction}

Two of the crucial signals of the Quark Gluon Plasma (QGP) - the most perfect fluid known to humanity - are collective flow and energy loss. Collective flow of long range correlations has been measured via the Fourier coefficients of the particle spectra i.e. $v_{n}\{m\}$ where $\mathrm{n}$ indicates the harmonic and $\mathrm{m}$ the number of particles correlated. While a finite $v_{2}\{m\}$ arises primarily from the geometry of the overlap between two heavy ions, the measured finite $v_{3}\{m\}$ exists because of the quantum mechanical fluctuations of the position of the nucleons in the overlap region [1,2]. Energy loss is measured via the nuclear modification factor $R_{A A}$ where the ratio of the particle spectra in a large AA system is normalized by the particle spectra in a pp collision all normalized by the number of binary collisions. When no energy loss is experienced $R_{A A} \rightarrow 1$ otherwise for a suppression $R_{A A}<1$ (for a review see [3]).

The dynamics of the soft sector of the strongly interacting soup of quarks and gluons can be described very well via event-by-event relativistic viscous hydrodynamical models that assume an almost vanishing shear viscosity to entropy density ratio [4, 5, 6, 7, 8, 9]. For the hard and heavy flavor sector additional energy loss mechanisms are needed in order to describe the suppression of both high transverse momentum, $p_{T} \gtrsim 10 \mathrm{GeV}$ and heavy quarks particles as they lose energy traveling through the dense QGP. Additionally, it was found in recent years that realistic hydrodynamical medium was required in order to describe the azimuthal anistropies of these hard probes $[10,11]$ (especially $v_{3}$ and multiparticle cumulants [12]).

When the QGP was first discovered, heavy AA collisions were explored because it was thought that only a large system would be able to produce the QGP. However, in recent years it has been found by a variety of experiments $[13,14,15,16,17,18,19,20,21,22,23,24,25,26,27,28,29$, $30,31,32,33$ ] signals for the QGP in small systems such as pPb, dAu, ${ }^{3} \mathrm{HeAu}, \mathrm{pAu}$, and possibly even $\mathrm{pp}$, which have been reasonably well reproduced and predicted by relativistic hydrodynamics $[34,35,36,37,38,39,40,41,42]$ (although a number of questions still remain). At the same time in small systems the nuclear modification factor was equivalent with 1 [43], which implied that no energy loss was seen or that the definitions of energy loss had to be refined in small systems. However, a surprising recent result was then released by the CMS collaboration in $\mathrm{pPb}$ collisions where they found that the heavy flavor sector still produced a significant $v_{2}$ but that a suppression was seen compared to other lighter particles [16].

Because of these recent results from CMS, we use D mesons as a test bed for energy loss in small systems. In this proceedings we explore the sensitivity of D mesons to the system size, specifically making the comparison between $\mathrm{PbPb}$ and $\mathrm{XeXe}$ collisions, which were recently ran at the LHC. However, XeXe collisions provided a surprise beyond system size effects. Assuming a spherical Xenon nucleus, hydrodynamic predictions of $v_{2}\{2\}$ in central collision for the ratio of $\mathrm{XeXe} / \mathrm{PbPb}$ collision significantly under-predicted the experimental data [7, 6], however, the inclusion of a deformed Xenon nucleus shifted the theoretical predictions closer to the experimental data [7]. Since this is an effect that is only seen in central collisions, a $\mathrm{PbPb}$ to $\mathrm{XeXe}$ comparison allows a test of both system size dependence (in the $30-50 \%$ centrality class) and the sensitivity to a deformed nucleus in the heavy flavor sector (in the $0-10 \%$ centrality class). 


\section{Model}

In recent years it was found that a more realistic description of the QGP medium is a necessary tool for simultaneously reproducing the nuclear modification factor $R_{A A}$ and the high $p_{T}$ and heavy flavor azimuthal anisotropies $v_{n}\{m\}\left(p_{T}\right)[44,10,11,45]$. Therefore we include a realistic event-by-event description of the medium coupled to modular heavy flavor code using Trento+v-USPhydro+DAB-MOD. The initial conditions are generated using Trento [46] where $p=0, \sigma=0.51 \mathrm{fm}$, and $k=1.6$ for $\mathrm{PbPb} 5.02 \mathrm{TeV}$ and for XeXe 5.44 TeV (note for Xenon we use the deformed Wood-Saxon parameters described in [47, 7]). The hydrodynamic model is v-USPhydro [48, 49, 50] where we use the same parameters for LHC run 2 as in [51] where $\tau_{0}=0.6 \mathrm{fm}, \eta / s \sim 0.05$, and $T_{F O}=150 \mathrm{MeV}$. To describe the heavy flavor sector we run DABMOD [45] a modular Monte Carlo simulation that samples heavy quarks using distributions from pQCD FONLL calculations [52, 53], implements either a parameterized energy loss model+energy loss fluctuations or a Langevin model, and then this is followed by fragmentation functions and coalescence to obtain the final particle yields.

In order to make predictions for smaller systems size we hold all our parameters fixed based on the $\mathrm{PbPb} 5.02 \mathrm{TeV}$ results, which have already been well-tested compared to experimental data [54]. Then the system size dependence arises through the initial conditions+hydrodynamical backgrounds calculated within Trento+v-USPhydro that were already discussed in detail in [55].

\section{Deformed Xe}

The geometry of the initial conditions can be quantified using the vector quantities of eccentricities

$$
\mathscr{E}_{n}=\frac{\int r^{n} e^{i n \phi} \rho(r, \phi) r d r d \phi}{\int r^{n} \rho(r, \phi) r d r d \phi},
$$

which are very strongly correlated with the final flow harmonics vector on an event-by-event basis in the soft sector $[56,57,58,59,60,61]$, high $p_{T}$ all charged particles [11, 10], and the heavy flavor sector $[44,45]$. We will mostly be focusing on only the magnitude of the eccentricities, $\varepsilon_{n}$, in this paper due to the changes it experiences for deformed nucleus as well as system size dependence.

In Fig. 1 we compare the effect of the ratio of the eccentricities in $\mathrm{XeXe}$ to $\mathrm{PbPb}$ for $\varepsilon_{2}$ and $\varepsilon_{3}$. For spherical AA collisions we generally expect that in central collisions $\varepsilon_{2}$ and $\varepsilon_{3}$ are inversely proportional to the system size (see Fig. 10 from [55]). Since the radius of Xenon is smaller than Lead we generally expect that the $v_{n}$ 's will also be larger, which is seen in the right plot in Fig. 1 for both the experiment and theory. However, what is also clear is that the prediction for a spherical Xenon nucleus significantly underpredicts the experimental data for $v_{2}^{X e}\{2\} / v_{2}^{P b}\{2\}$ in central collisions. The theoretical prediction for a deformed Xenon nucleus is closer to experimental data (CMS [62], ALICE [63], and ATLAS [64]) but still slightly somewhat undepredicts the effect [65], it may be that a larger deformation is required or that other nuclear structure effects are missing from heavy-ion initial condition models. Finally, it should be clear that the effect of the deformation is only relevant in central collisions. For more peripheral collisions the dominating geometrical effect comes from the impact region itself.

In Fig. 2 we compare $v_{2}\{2\}\left(p_{T}\right)$ and $v_{3}\{2\}\left(p_{T}\right)$ from $\mathrm{PbPb} 5.02 \mathrm{TeV}$ collisions to both a spherical Xenon nucleons versus a prolate Xenon for XeXe $5.44 \mathrm{TeV}$ collisions in the $0-10 \%$ 




Figure 1: On the left, ratio of the eccentricities of $\mathrm{Pb} \mathrm{Pb} / \mathrm{Xe} \mathrm{Xe}$ collisions at the LHC calculated using TRENTO [46]. Figure taken from [7]. On the right, theory predictions from trento+v-USPhydro with and without a deformed Xe nucleus compare to experimental data from [64]

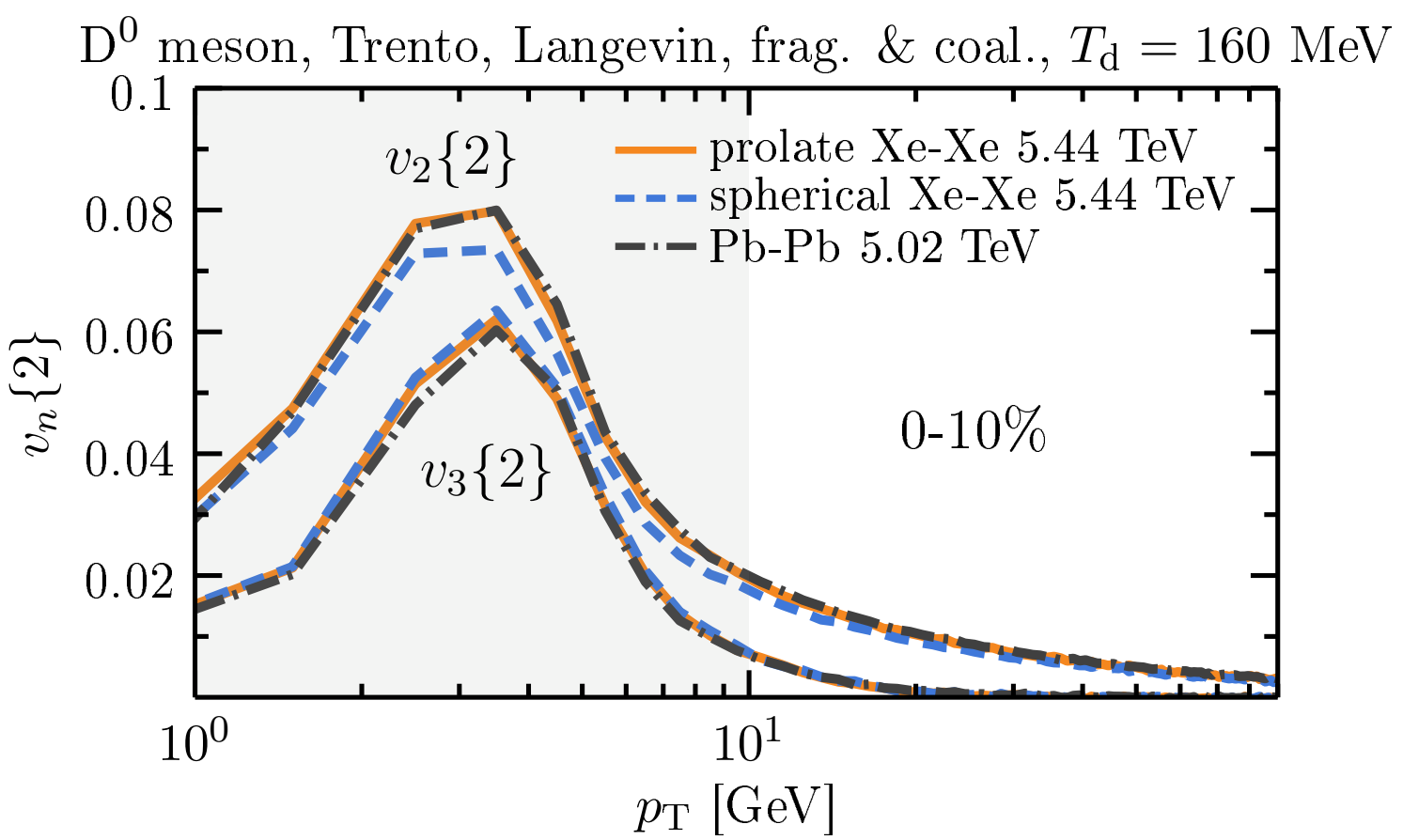

Figure 2: $v_{n}\{2\}\left(p_{T}\right)$ of $\mathrm{D}$ mesons in $0-10 \% \mathrm{PbPb}$ vs. XeXe collisions compared using Trento+vUSPhydro+DAB-MOD.

centrality class. We find a small enhancement in $v_{3}\{2\}$, which is consistent with the soft sector results from $[7,55]$. This enhancement arises due to the universal scaling of $v_{3}\{2\}$ with Npart as demonstrated in Fig. 17 from [55]. Since $v_{3}\{2\}$ has a maximum in $N_{\text {part }} \approx 100$ and central XeXe collisions have an $N_{\text {part }} \approx 200-250$ compared to $\mathrm{PbPb}$ 's $N_{\text {part }} \approx 310-415$ then one expects a 
continually growing $v_{3}\{2\}$ as $N_{\text {part }} \rightarrow 100$. We note that the $v_{3}\{2\}$ result has nothing to do with a deformed nucleus since both a prolate and spherical nucleus are in agreement.

More interestingly, however, is $v_{2}\{2\}$ that shows a clear enhancement for a prolate Xenon nucleus whereas for a spherical Xenon nucleus one predicts that it should be suppressed at intermediate $p_{T}$ 's. This is a quite interesting result because it is not even happening in just the ultracentral collisions where the original XeXe effect of deformation was most sensitive [7] but rather in the $0-10 \%$ centrality class. Additionally, it appears that intermediate $p_{T}\left(p_{T}=2-5 \mathrm{GeV}\right)$ is the most sensitive to the deformation, which is also surprising.

\section{System size: $\mathrm{PbPb}$ vs. XeXe collisions}



Figure 3: $v_{n}\{2\}\left(p_{T}\right)$ of $\mathrm{D}$ mesons in $30-50 \% \mathrm{~Pb} \mathrm{~Pb}$ vs. Xe Xe collisions compared using Trento+vUSPhydro+DAB-MOD.

Returning to our original purpose of studying the system size dependence of D mesons $v_{n}\{2\}\left(p_{T}\right)$ we now compare $\mathrm{PbPb}$ collisions to XeXe collisions. In Fig. 1 it was shown that the eccentricties are identical for the spherical and deformed Xenon nucleus for any collisions in centralities more peripheral than $\sim 20 \%$. Thus, exploring comparisons in the $30-50 \%$ centrality class is certainly safe from deformation effects. Indeed, in Fig. 3 we compare both $v_{2}\{2\}\left(p_{T}\right)$ and $v_{3}\{2\}\left(p_{T}\right)$ from $\mathrm{PbPb}$ collisions and $\mathrm{XeXe}$ collisions and see that there is no significant difference between the prolate and spherical Xenon nucleus.

From the eccentricities shown in Fig. 1 we expect that $v_{2}^{P b}\{2\}\left(p_{T}\right)>v_{2}^{X e}\{2\}\left(p_{T}\right)$ and that $v_{3}^{P b}\{2\}\left(p_{T}\right) \lesssim v_{3}^{X e}\{2\}\left(p_{T}\right)$, which is what is found in the soft sector [65]. While the prediction from the eccentricities for $v_{2}$ holds for D mesons, we find that $v_{3}^{P b}\{2\}\left(p_{T}\right)>v_{3}^{X e}\{2\}\left(p_{T}\right)$, which is in contrast to both the eccentricities predictions and what is seen in the soft sector. There are 
a number of reasons why this may occur, for instance, the higher decoupling temperature that we use in the heavy flavor sector or the possibility that there is a nontrivial interplay between $v_{3}$ with the physics of the heavy flavor sector i.e. energy loss, fragmentation functions, or coalescence. However, we also note that this suppression of the expected $v_{n}$ 's in smaller systems is consistent with what $\mathrm{CMS}$ found comparing $\mathrm{pPb}$ to $\mathrm{PbPb}$ collisions. Thus, we are encouraged that the heavy flavor sector can provide new information on the question of energy loss in small systems.

\section{Conclusions}

In conclusion, we have made the first event-by-event azimuthal anisotropies predictions in $\mathrm{XeXe} 5.44 \mathrm{TeV}$ collisions (note previous predictions were made for XeXe collision on top of a non-fluctuating background using a Bjoerken expansion in [66, 67]). We find that surprisingly enough that $\mathrm{D}$ mesons are sensitive to a deformed ${ }^{129} \mathrm{Xe}$ in the $0-10 \%$ centrality class and that this effect is enhanced in the range of $p_{T} \sim 3-5 \mathrm{GeV}$. From this we conclude that it would be very interesting for experimentalists to compare $\mathrm{D}$ meson azimuthal anisotropies in deformed AA collisions (such as Uranium or the upcoming isobar run) as well.

In mid-central collisions of $30-50 \%$ centrality we have shown that there is a suppression of the $v_{n}\{2\}\left(p_{T}\right)$ 's due to system size effects. From the eccentricities alone, one would expect a small suppression on the order of a few percentage points such that $v_{2}^{X e}\{2\}<v_{2}^{P b}\{2\}$ in midcentral collisions but that cannot explain the over 20\% suppression of D mesons $v_{2}^{X e}\{2\}$ compared to $v_{2}^{P b}\{2\}$. Thus it appears that $\mathrm{D}$ mesons are more sensitive to system size effects.

We have not yet checked the sensitivity to other medium parameters such as $\tau_{0}[68]$ and $\eta / s$ in peripheral collisions [11]. It also remains to be seen if the hard and heavy flavor sector are sensitive to the equation of state as explored in [51], which could be connected to the initialization time as well.

\section{Acknowledgments}

The authors thank Fundação de Amparo à Pesquisa do Estado de São Paulo (FAPESP) and Conselho Nacional de Desenvolvimento Científico e Tecnológico (CNPq) for support. C.A.G.P. is supported by the NSFC under grant No. 11521064, MOST of China under Project No. 2014CB845404. J.N.H. acknowledges the support of the Alfred P. Sloan Foundation, support from the US-DOE Nuclear Science Grant No. DE-SC0019175, and the Office of Advanced Research Computing (OARC) at Rutgers, The State University of New Jersey for providing access to the Amarel cluster and associated research computing resources that have contributed to the results reported here. JN is partially supported by CNPq grant 306795/2017-5 and FAPESP grant 2017/05685-2.

\section{References}

[1] B. Alver and G. Roland, Collision geometry fluctuations and triangular flow in heavy-ion collisions, Phys. Rev. C81 (2010) 054905 [1003.0194].

[2] J. Takahashi, B. M. Tavares, W. L. Qian, R. Andrade, F. Grassi, Y. Hama et al., Topology studies of hydrodynamics using two particle correlation analysis, Phys. Rev. Lett. 103 (2009) 242301 [0902.4870]. 
[3] P. Jacobs and X.-N. Wang, Matter in extremis: Ultrarelativistic nuclear collisions at RHIC, Prog. Part. Nucl. Phys. 54 (2005) 443 [hep-ph/ 0405125 ].

[4] H. Niemi, K. J. Eskola, R. Paatelainen and K. Tuominen, Predictions for 5.023 $\mathrm{TeV} \mathrm{Pb}+\mathrm{Pb}$ collisions at the CERN Large Hadron Collider, Phys. Rev. C93 (2016) 014912 [1511. 04296 ].

[5] J. Noronha-Hostler, M. Luzum and J.-Y. Ollitrault, Hydrodynamic predictions for 5.02 TeV Pb-Pb collisions, Phys. Rev. C93 (2016) 034912 [1511. 06289 ].

[6] K. J. Eskola, H. Niemi, R. Paatelainen and K. Tuominen, Predictions for multiplicities and flow harmonics in $5.44 \mathrm{TeV}$ Xe+Xe collisions at the CERN Large Hadron Collider, Phys. Rev. C97 (2018) 034911 [1711.09803].

[7] G. Giacalone, J. Noronha-Hostler, M. Luzum and J.-Y. Ollitrault, Hydrodynamic predictions for 5.44 TeV Xe+Xe collisions, Phys. Rev. C97 (2018) 034904 [1711.08499].

[8] C. Gale, S. Jeon, B. Schenke, P. Tribedy and R. Venugopalan, Event-by-event anisotropic flow in heavy-ion collisions from combined Yang-Mills and viscous fluid dynamics, Phys. Rev. Lett. 110 (2013) 012302 [1209.6330].

[9] J. E. Bernhard, J. S. Moreland, S. A. Bass, J. Liu and U. Heinz, Applying Bayesian parameter estimation to relativistic heavy-ion collisions: simultaneous characterization of the initial state and quark-gluon plasma medium, Phys. Rev. C94 (2016) 024907 [1605.03954].

[10] J. Noronha-Hostler, B. Betz, J. Noronha and M. Gyulassy, Event-by-event hydrodynamics + jet energy loss: A solution to the $R_{A A} \otimes v_{2}$ puzzle, Phys. Rev. Lett. 116 (2016) 252301 [1602 . 03788 ].

[11] B. Betz, M. Gyulassy, M. Luzum, J. Noronha, J. Noronha-Hostler, I. Portillo et al., Cumulants and nonlinear response of high $p_{T}$ harmonic flow at $\sqrt{s_{N N}}=5.02$ TeV, Phys. Rev. C95 (2017) 044901 [1609.05171].

[12] CMS collaboration, Azimuthal anisotropy of charged particles with transverse momentum up to 100 GeV/c in PbPb collisions at $\sqrt{s}_{N N}=5.02$ TeV, Phys. Lett. B776 (2018) 195 [1702.00630].

[13] ATLAS collaboration, Measurement of multi-particle azimuthal correlations in $p p, p+P b$ and low-multiplicity Pb+Pb collisions with the ATLAS detector, Eur. Phys. J. C77 (2017) 428 [1705.04176].

[14] ATLAS collaboration, Measurement of long-range multiparticle azimuthal correlations with the subevent cumulant method in pp and $p+P b$ collisions with the ATLAS detector at the CERN Large Hadron Collider, Phys. Rev. C97 (2018) 024904 [1708.03559].

[15] ATLAS collaboration, Measurement with the ATLAS detector of multi-particle azimuthal correlations in p+Pb collisions at $\sqrt{s_{N N}}=5.02$ TeV, Phys. Lett. B725 (2013) 60 [1303.2084].

[16] CMS collaboration, Elliptic flow of charm and strange hadrons in high-multiplicity pPb collisions at $\sqrt{s_{\mathrm{NN}}}=8.16 \mathrm{TeV}$, Phys. Rev. Lett. 121 (2018) 082301 [1804.09767].

[17] CMS collaboration, Multiplicity and transverse momentum dependence of two-and four-particle correlations in pPb and PbPb collisions, Phys. Lett. B724 (2013) 213 [1305. 060 9].

[18] CMS collaboration, Long-range two-particle correlations of strange hadrons with charged particles in pPb and PbPb collisions at LHC energies, Phys. Lett. B742 (2015) 200 [1409. 3392].

[19] CMS collaboration, Evidence for Collective Multiparticle Correlations in p-Pb Collisions, Phys. Rev. Lett. 115 (2015) 012301 [1502.05382]. 
[20] CMS collaboration, Evidence for transverse momentum and pseudorapidity dependent event plane fluctuations in PbPb and pPb collisions, Phys. Rev. C92 (2015) 034911 [1503.01692].

[21] CMS collaboration, Observation of Correlated Azimuthal Anisotropy Fourier Harmonics in pp and p+Pb Collisions at the LHC, Phys. Rev. Lett. 120 (2018) 092301 [1709.09189].

[22] ALICE collaboration, Long-range angular correlations of $\pi, K$ and $p$ in $p$-Pb collisions at $\sqrt{s_{\mathrm{NN}}}=$ 5.02 TeV, Phys. Lett. B726 (2013) 164 [1307. 3237].

[23] ALICE collaboration, Multiparticle azimuthal correlations in $\mathrm{p}-\mathrm{Pb}$ and $\mathrm{Pb}-\mathrm{Pb}$ collisions at the CERN Large Hadron Collider, Phys. Rev. C90 (2014) 054901 [1406.2474].

[24] PHENIX collaboration, Quadrupole Anisotropy in Dihadron Azimuthal Correlations in Central $d+A u$ Collisions at $\sqrt{s_{N N}}=200$ GeV, Phys. Rev. Lett. 111 (2013) 212301 [1303.1794].

[25] PHENIX collaboration, Measurement of long-range angular correlation and quadrupole anisotropy of pions and (anti)protons in central $d+$ Au collisions at $\sqrt{s_{N N}}=200 \mathrm{GeV}$, Phys. Rev. Lett. 114 (2015) $192301[1404.7461]$.

[26] PHENIX collaboration, Creating small circular, elliptical, and triangular droplets of quark-gluon plasma, 1805.02973.

[27] PHENIX collaboration, Pseudorapidity dependence of particle production and elliptic flow in asymmetric nuclear collisions of $p+A l, p+A u, d+A u$, and ${ }^{3} \mathrm{He}+A u$ at $\sqrt{s_{N N}}=200 \mathrm{GeV}$, 1807.11928.

[28] PHENIX collaboration, Measurements of elliptic and triangular flow in high-multiplicity ${ }^{3} \mathrm{He}+\mathrm{Au}$ collisions at $\sqrt{s_{N N}}=200 \mathrm{GeV}$, Phys. Rev. Lett. 115 (2015) 142301 [1507.06273].

[29] C. Aidala et al., Measurement of long-range angular correlations and azimuthal anisotropies in high-multiplicity $p+$ Au collisions at $\sqrt{s_{N N}}=200 \mathrm{GeV}$, Phys. Rev. C95 (2017) 034910 [1609.02894].

[30] PHENIX collaboration, Measurements of mass-dependent azimuthal anisotropy in central $p+A u$, $d+A u$, and ${ }^{3} \mathrm{He}+\mathrm{Au}$ collisions at $\sqrt{s_{N N}}=200 \mathrm{GeV}$, Phys. Rev. C97 (2018) 064904 [1710.09736].

[31] PHENIX collaboration, Measurement of emission angle anisotropy via long-range angular correlations with high $p_{T}$ hadrons in $d+A u$ and $p+p$ collisions at $\sqrt{s_{N N}}=200$ GeV, Phys. Rev. C98 (2018) 014912 [1711.09003].

[32] PHENIX collaboration, Measurements of azimuthal anisotropy and charged-particle multiplicity in $d+$ Au collisions at $\sqrt{s_{N N}}=200,62.4,39$, and $19.6 \mathrm{GeV}$, Phys. Rev. C96 (2017) 064905 [1708.06983].

[33] PHENIX collaboration, Measurements of Multiparticle Correlations in d Au Collisions at 200, 62.4, 39, and 19.6 GeV and p+ Au Collisions at $200 \mathrm{GeV}$ and Implications for Collective Behavior, Phys. Rev. Lett. 120 (2018) 062302 [1707.06108].

[34] P. Bozek, Collective flow in p-Pb and d-Pd collisions at TeV energies, Phys. Rev. C85 (2012) 014911 [1112.0915].

[35] P. Bozek and W. Broniowski, Correlations from hydrodynamic flow in p-Pb collisions, Phys. Lett. B718 (2013) 1557 [1211.0845].

[36] P. Bozek, W. Broniowski and G. Torrieri, Mass hierarchy in identified particle distributions in proton-lead collisions, Phys. Rev. Lett. 111 (2013) 172303 [1307. 5060 ]. 
[37] P. Bozek and W. Broniowski, Collective dynamics in high-energy proton-nucleus collisions, Phys. Rev. C88 (2013) 014903 [1304. 304 4].

[38] I. Kozlov, M. Luzum, G. Denicol, S. Jeon and C. Gale, Transverse momentum structure of pair correlations as a signature of collective behavior in small collision systems, 1405.3976.

[39] Y. Zhou, X. Zhu, P. Li and H. Song, Investigation of possible hadronic flow in $\sqrt{s_{N N}}=5.02 \mathrm{TeV}$ p-Pb collisions, Phys. Rev. C91 (2015) 064908 [1503.06986].

[40] W. Zhao, Y. Zhou, H. Xu, W. Deng and H. Song, Hydrodynamic collectivity in proton-proton collisions at 13 TeV, Phys. Lett. B780 (2018) 495 [1801.00271].

[41] H. Mäntysaari, B. Schenke, C. Shen and P. Tribedy, Imprints of fluctuating proton shapes on flow in proton-lead collisions at the LHC, Phys. Lett. B772 (2017) 681 [1705.03177].

[42] R. D. Weller and P. Romatschke, One fluid to rule them all: viscous hydrodynamic description of event-by-event central $p+p, p+P b$ and $P b+P b$ collisions at $\sqrt{s}=5.02 \mathrm{TeV}$, Phys. Lett. B774 (2017) $351[1701.07145]$.

[43] CMS collaboration, Charged-particle nuclear modification factors in $\mathrm{PbPb}$ and $\mathrm{pPb}$ collisions at $\sqrt{s_{\mathrm{N} \mathrm{N}}}=5.02 \mathrm{TeV}, \mathrm{JHEP} 04$ (2017) 039 [1611.01664].

[44] M. Nahrgang, J. Aichelin, S. Bass, P. B. Gossiaux and K. Werner, Elliptic and triangular flow of heavy flavor in heavy-ion collisions, Phys. Rev. C91 (2015) 014904 [1410. 5396].

[45] C. A. G. Prado, J. Noronha-Hostler, R. Katz, A. A. P. Suaide, J. Noronha, M. G. Munhoz et al., Event-by-event correlations between soft hadrons and $\mathrm{D}^{0}$ mesons in $5.02 \mathrm{TeV} \mathrm{PbPb}$ collisions at the CERN Large Hadron Collider, Phys. Rev. C96 (2017) 064903 [1611. 02965 ].

[46] J. S. Moreland, J. E. Bernhard and S. A. Bass, Alternative ansatz to wounded nucleon and binary collision scaling in high-energy nuclear collisions, Phys. Rev. C92 (2015) 011901 [1412 . 4708 ].

[47] P. Moller, A. J. Sierk, T. Ichikawa and H. Sagawa, Nuclear ground-state masses and deformations: FRDM(2012), Atom. Data Nucl. Data Tabl. 109-110 (2016) 1 [1508. 06294 ].

[48] J. Noronha-Hostler, G. S. Denicol, J. Noronha, R. P. G. Andrade and F. Grassi, Bulk Viscosity Effects in Event-by-Event Relativistic Hydrodynamics, Phys. Rev. C88 (2013) 044916 [1305. 1981 ].

[49] J. Noronha-Hostler, J. Noronha and F. Grassi, Bulk viscosity-driven suppression of shear viscosity effects on the flow harmonics at energies available at the BNL Relativistic Heavy Ion Collider, Phys. Rev. C90 (2014) 034907 [1406.3333].

[50] J. Noronha-Hostler, J. Noronha and M. Gyulassy, Sensitivity of flow harmonics to subnucleon scale fluctuations in heavy ion collisions, Phys. Rev. C93 (2016) 024909 [1508 . 02455 ].

[51] P. Alba, V. Mantovani Sarti, J. Noronha, J. Noronha-Hostler, P. Parotto, I. Portillo Vazquez et al., Effect of the QCD equation of state and strange hadronic resonances on multiparticle correlations in heavy ion collisions, Phys. Rev. C98 (2018) 034909 [1711.05207].

[52] M. Cacciari, M. Greco and P. Nason, The P(T) spectrum in heavy flavor hadroproduction, JHEP 05 (1998) 007 [hep-ph/9803400].

[53] M. Cacciari, S. Frixione and P. Nason, The $p(T)$ spectrum in heavy flavor photoproduction, JHEP 03 (2001) 006 [hep-ph/ 0102134$].$

[54] R. Katz, C. A. G. Prado, J. Noronha-Hostler, A. A. P. Suaide, J. Noronha and M. G. Munhoz, Heavy-flavor dynamics in event-by-event viscous hydrodynamic backgrounds, PoS HardProbes2018 (2018) 030 [1812.08009]. 
[55] M. D. Sievert and J. Noronha-Hostler, Shrinking the Quark Gluon Plasma, 1901.01319.

[56] D. Teaney and L. Yan, Triangularity and Dipole Asymmetry in Heavy Ion Collisions, Phys. Rev. C83 (2011) 064904 [1010.1876].

[57] F. G. Gardim, F. Grassi, M. Luzum and J.-Y. Ollitrault, Mapping the hydrodynamic response to the initial geometry in heavy-ion collisions, Phys. Rev. C85 (2012) 024908 [1111. 6538].

[58] H. Niemi, G. S. Denicol, H. Holopainen and P. Huovinen, Event-by-event distributions of azimuthal asymmetries in ultrarelativistic heavy-ion collisions, Phys. Rev. C87 (2013) 054901 [1212.1008].

[59] D. Teaney and L. Yan, Non linearities in the harmonic spectrum of heavy ion collisions with ideal and viscous hydrodynamics, Phys. Rev. C86 (2012) 044908 [1206.1905].

[60] Z. Qiu and U. W. Heinz, Event-by-event shape and flow fluctuations of relativistic heavy-ion collision fireballs, Phys. Rev. C84 (2011) 024911 [1104.0650].

[61] F. G. Gardim, J. Noronha-Hostler, M. Luzum and F. Grassi, Effects of viscosity on the mapping of initial to final state in heavy ion collisions, Phys. Rev. C91 (2015) 034902 [1411.2574].

[62] CMS collaboration, Charged particle angular correlations in XeXe collision at $\sqrt{s_{N N}}=5.44 \mathrm{TeV}$, .

[63] ALICE collaboration, Anisotropic flow in Xe-Xe collisions at $\sqrt{\mathbf{s}_{\mathrm{NN}}}=\mathbf{5 . 4 4}$ TeV, Phys. Lett. B784 (2018) 82 [1805.01832].

[64] ATLAS collaboration, Measurement of the azimuthal anisotropy of charged particle production in $\mathrm{Xe}+\mathrm{Xe}$ collisions at $\sqrt{s_{\mathrm{NN}}}=5.44 \mathrm{TeV}$ with the ATLAS detector, (Geneva), CERN, CERN, 2018.

[65] G. Giacalone, J. Noronha-Hostler, M. Luzum and J.-Y. Ollitrault, Confronting hydrodynamic predictions with Xe-Xe data, in 27th International Conference on Ultrarelativistic Nucleus-Nucleus Collisions (Quark Matter 2018) Venice, Italy, May 14-19, 2018, 2018, 1807.05557.

[66] D. Zigic, I. Salom, J. Auvinen, M. Djordjevic and M. Djordjevic, DREENA-B framework: first predictions of $R_{A A}$ and $v_{2}$ within dynamical energy loss formalism in evolving QCD medium, Phys. Lett. B791 (2019) 236 [1805.04786].

[67] M. Djordjevic, D. Zigic, M. Djordjevic and J. Auvinen, How to test path-length dependence in energy loss mechanisms: analysis leading to a new observable, 1805.04030.

[68] C. Andres, N. Armesto, H. Niemi, R. Paatelainen and C. A. Salgado, Jet quenching as a probe of the initial stages in heavy-ion collisions, 1902.03231. 